\title{
THE IONIZATION POTENTIAL OF ELECTRODES IN VARIOUS GASES.
}

By F. M. BISHOP.

THE $\mathrm{HE}$ object of the present investigation was to redetermine the ionizing potential of certain gases under different experimental conditions and to extend the work to some simple compounds and see if the combination of one atom with another had any effect on its ionizing potential.

Two forms of apparatus have been used heretofore for the direct measurement of ionization potentials; one in which the source of electrons was a plate illuminated by ultra-violet light, was used by Lenard ${ }^{1}$ and by Dember, ${ }^{2}$ and the other form in which electrons were liberated from a hot wire or Wehnelt cathode was used by von Baeyer, ${ }^{3}$ Franck and Hertz ${ }^{4}$ and Pawlow. ${ }^{5}$ Since this work was in progress an important modification of this second source has been described by Goucher. ${ }^{6}$

The results obtained by these observers with the two forms of apparatus differ greatly, and it seemed desirable to find the reason for this discrepancy by employing both methods of liberating the electrons and using an apparatus in which the important quantities could be varied at will.

The object in view in finding the ionizing potential of a simple compound, the ionizing potential of whose components are known, was, first, to determine whether the ionizing potential is an atomic property and not dependent on the molecular combination; and second, if this first proved to be the case, to open the possibility of determining the ionizing potentials of some substances, which in their simple uncombined state do not lend themselves easily to this direct method of determination. Naturally the method would still be applicable only to substances in which the unknown component ionized at the lower potential.

Apparatus and Method.-The method is essentially that described by Franck and Hertz, Pawlow, and Goucher in which plate electrodes are used.

1 P. Lenard, Ann d. Phys. (4), 8, I49, I902.

2 H. Dember, Ann d. Phys. (4), 30, I37, I909.

3 O. V. Baeyer, Verh d. D. Phys. Ges., xo, 96, 1908.

${ }^{4}$ Franck and Hertz, Deutsch. Phys. Ges., Vol. I5, p. 34, I9 3.

5 Pawlow, Proc. Roy. Soc., Vol. 90, p. 390, r9r4.

${ }^{6}$ Goucher, Phys. Rev., Vol. 8, p. 56I, I9I6. 
The diagram of Fig. I shows the apparatus drawn to scale. $A$ is a hot wire source of electrons which could be readily changed, $B$ a gauze to which an accelerating potential is applied, and $C$ a receiving electrode made of oxidized brass which was found to be insensitive photo-electrically. $B$ is attached to a metal cylinder $E$ and gauze screen $F$, which completely enclose and shield the ionization chamber from any charges that may accumulate on the glass walls of the containing vessel. $G$ and $H$ are inlet and outlet tubes respectively through which a continuous flow of gas is maintained during a set of readings by keeping a diffusion pump running at one end and allowing the gas to diffuse through a small capillary from a chamber in which the pressure could be suitably regulated at will. On either side of the main apparatus were liquid air traps to keep mercury vapor and also any vapor from the stopcock grease away from the ionization chamber. Immediately beyond the liquid air trap on the pump side was a McLeod gauge, and a discharge tube similar to the one described by Pawlow in which the pressure could be suitably regulated and the purity of the gas tested with a direct vision spectroscope.

The apparatus in which photo-electrons were used differed from the one described in the following particulars, which are indicated in Fig. I bK

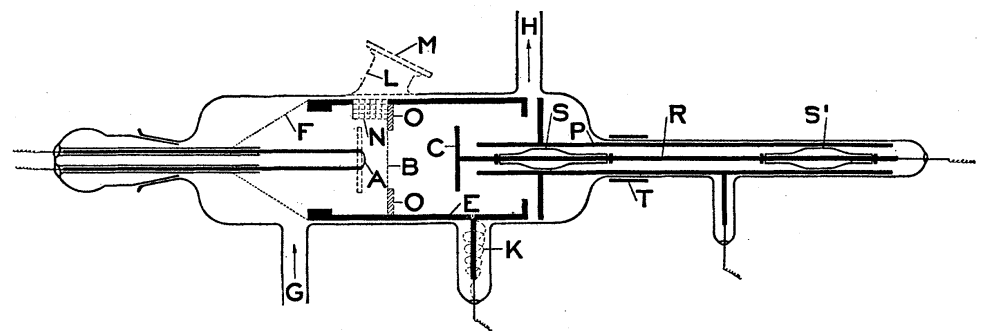

Fig. 1.

dotted lines. An aluminum plate was substituted for the hot wire $A$, the apparatus contained a side tube $L$ and quartz window $M$, and opening $N$ in the metal cylinder, so that a source of ultra-violet light could be focused on the aluminum plate. The cylinder $E$ could be moved by means of an iron ring by a magnet placed outside the tube. A fine copper wire in the form of a coil was substituted for the rigid contact $K$. This tube contained a metal ring $O$ inserted as shown in Fig. I to shield the electrode $C$ from scattered ultra-violet light as much as possible. Ultra-violet light striking $C$, with the surrounding metal part $E$ at a higher potential, will cause photo-electrons to be given off from $C$ and it will be noted that the resultant charging up of the electrometer is the 
same sign as that due to the ionization we seek to measure. $C$ was made of oxidized brass to minimize this effect. Control readings were taken with $A$ and $B$ at the potential of $B$ and the positive deflections due to this photo-electric effect subtracted from the ionization deflections. The object in having the cylinder $E$ movable was to be able to vary the distance between $A$ and $B$ through which the electrons were accelerated, and to set this distance less than the mean free path of an electron at any pressure used.

Preliminary work in hydrogen made with this apparatus using ultraviolet light showed that the form and position of the curve were not affected by change of pressure, except when the distance $A B$ was made comparatively long and the pressure high enough so that additional electrons would be given off due to ionization in the region $A B$ and these electrons in turn could acquire energy enough to ionize. This, it will be observed, could not affect the form of the curve until the applied accelerating potential was twice the minimum potential required to ionize.

Since the relative distances had been shown to have no effect on the ionizing potential by work with the first form of apparatus, the construction of the second piece of apparatus, in which the hot cathode was used, was considerably simplified. A rigid contact was substituted at $K$ for the flexible one previously employed and the iron ring was done away with.

The distance between $A$ and $C$ in each case was $4 \mathrm{~cm}$. and in the hotwire apparatus the distance between $A$ and $B$ was $\mathrm{I} \mathrm{cm}$. The cylinder $E$ was of brass and also the gauze $B$, the mesh of which was about $\mathrm{I} \mathrm{mm}$.

The writer wishes here to express his thanks to Mr. A. Greiner, of the firm of Green and Bauer, who made both pieces of apparatus.

The electrometer used was of the Dolezalek type, sensitive to about 4,000 scale divisions per volt. This could also be used in connection with a mica condenser which reduced the sensitiveness in the ratio of 9 to $\mathrm{I}$. The electrometer connection was shielded by an earthed metal screen $P$ insulated from the lead $R$ by quartz insulators $S$ and $S^{\prime}$. Surface leaks over the outside of the glass were prevented by an earthed metal foil $T$ moistened with a solution of calcium chloride.

During the process of obtaining an ionization curve the potential of $A$ was kept 4 or more volts higher than $C$, so no electrons from $A$ could reach $C$. The accelerating field $A B$ was varied by varying the potential on $B$ by means of storage cells and a potentiometer. Since the retarding field between $B$ and $C$ is greater than the accelerating field between $A$ and $B$ no deflection of the electrometer will be observed until the electrons acquire sufficient energy in the region $A B$ to cause ionization in the region 
$B C$. When this potential is reached positive ions are driven to the negative electrode $C$. This potential between $A$ and $B$ at which positive ions begin to collect on $C$ is taken as the minimum ionizing potential of the gas. If the value of the ionizing potential is sought with extreme accuracy corrections must be applied for two reasons as was pointed out by Franck and Hertz and by Goucher. There is a drop in potential of at least 6/10 volt between the two ends of the wire $A$ due to the heating current. Conduction of heat to the leads causes the ends of the wire to assume a much lower temperature than the middle. Each of these causes tends to make the velocities of the electrons unequal. For a very accurate determination of the ionizing potential the equi-potential equitemperature source described by Goucher is undoubtedly superior. This form of a source was not used in the present investigation because the experiments were well under way before the method was published.

The accelerating potential recorded throughout this paper is the one between $B$ and the lower potential end of $A$. On account of the initial velocity with which the electrons leave the hotter central portion of the wire, this voltage more nearly represents the energy of a large fraction of the electrons leaving $A$, and smaller corrections have to be applied than if the voltages between $B$ and the positive end of the wire were taken.

In order to determine the relative number of electrons coming off with any particular velocity, electron current readings were taken where a potential slightly below the ionizing potential was applied between $A$ and $B$ and successive retarding potentials applied between $B$ and $C$. These electron currents were then plotted against the difference between accelerating and retarding potentials and this curve was then graphically differentiated, and the tangents plotted against the corresponding voltages of the electron current curve. This gives a velocity distribution curve in which the ordinates are proportional to the number of electrons coming off with any particular velocity. After a number of electron current curves had been taken at any given pressure it was observed that these curves had their maximum slope approximately at zero volts; that is, when the accelerating potential applied between $A$ and $B$ was equal to the retarding potential applied between $B$ and $C$. Further it was observed that the position of maximum slope could be altered by changing the heating current. In order to make the correction, which would eventually have to be applied to the ionization curve, as small as possible the heating current in the hot wire source of electrons was so regulated by successive trials that the resulting electron current had its maximum slope at zero volts. The value of this maximum tangent was set equal to Ioo. The ordinates at other potentials on this curve represent the 
number of electrons coming off at these potentials on the same arbitrary scale.

Measurements in Hydrogen.- Hydrogen was prepared electrolytically, dried by passing over calcium chloride and phosphorus pentoxide and passed over heated copper in an electric furnace to free it from any trace of oxygen. The hydrogen then passed through a trap immersed in liquid air and into the ionization chamber.

The author wishes here to express his thanks to Professor Boltwood for his suggestions and assistance in the preparation of the different gases used.

With the photo-electric apparatus a break occurred in the curve for hydrogen at about 16 volts as shown in Curve $(g)$, Fig. 2, while with the hot wire source this break occurred at I I volts; Curve (a), Fig. 2, being typical for this source. Since the electron current from the hot wire was much larger than the current from the ultra-violet light source, readings were taken with the current from the hot wire very much reduced, and in this case curves similar to $(g)$ could be reproduced. A very intense ultra-violet light source, moreover, gave curves such as $(f)$, Fig. 2, where a break occurred below I6 volts but a sharp bend in the curve occurred at about 16 volts. Curves $(b)$ and $(c)$ were taken with intermediate electron currents. They show ionization beginning at I I volts and a sharp increase at about 15.7 volts. This fact shows that there is a second and more intense type of ionization which begins at this higher potential. Goucher and Davis, after being informed of this result, have recently confirmed it with their apparatus.

The lack of complete agreement of this second break in the curve for the two sources, 16 volts for one and 15.7 for the other, may be attributed to the fact that a considerable correction has to be applied to the curves $(f)$ and $(g)$. The electron current curves with the ultra-violet light source did not have their maximum slope where the accelerating potential between $A$ and $B$ was equal to the retarding potential between $B$ and $C$, but where the accelerating potential $A B$ exceeded $B C$ by several tenths of a volt, that is, some of the applied energy was used up in helping the electrons out of the plate, so that we may conclude that the two methods are in good agreement and that this new second type of ionization in hydrogen begins at about 15.7 volts.

The experiments in which a hot wire source of electrons was used give I I volts for the ionizing potential of the first type of ionization in hydrogen in agreement with the work of other observers. With the ultra-violet light source of electrons it is shown that the number of electrons is usually not sufficient to permit the ionizing potential of the first type to be measured, and in some cases its presence may not even be detected. 
The pressures in the work on hydrogen varied between .oor $\mathrm{mm}$. and $.03 \mathrm{~mm}$., and the point at which ionization began seemed entirely independent of the pressure within these limits.

Nitrogen.-Nitrogen was prepared by heating ammonium chloride and sodium nitrite, bubbled through sodium hydroxide solution to remove any carbon dioxide formed. It next passed over heated copper and heated copper oxide in order to remove oxygen and hydrogen. It was dried by passing through calcium chloride and phosphorus pentoxide, then passed through a trap immersed in liquid air into the ionization

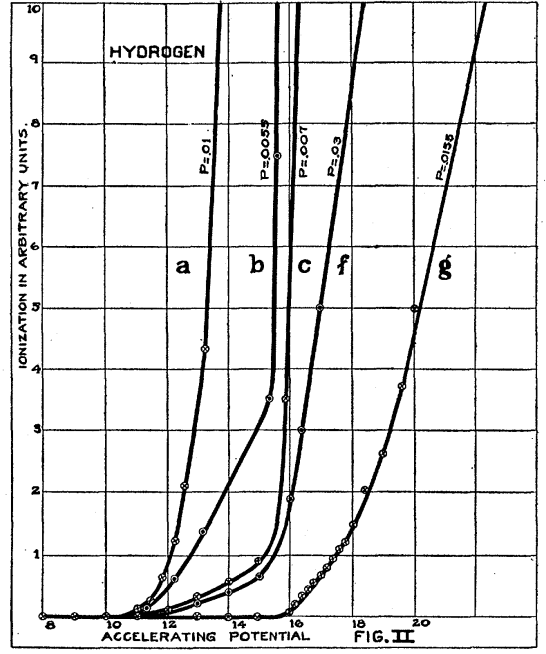

Fig. 2.

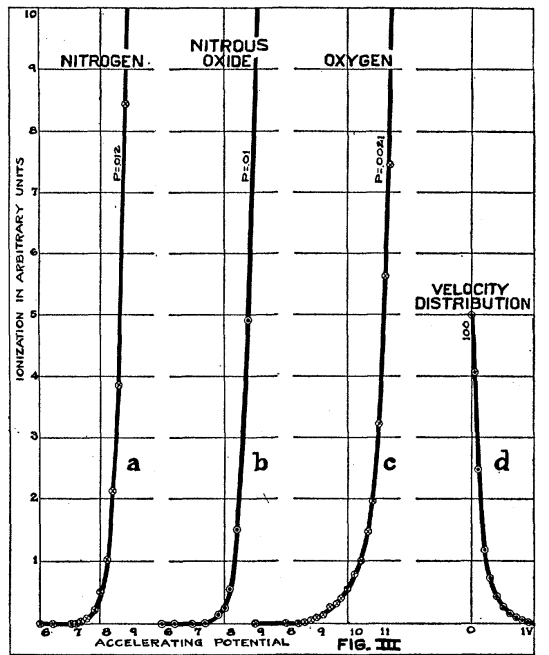

Fig. 3.

chamber. Curve (a), Fig. 3, is a typical curve for nitrogen, in which ionization begins at about 7.5 volts. This curve was taken at a pressure of .ol2 $\mathrm{mm}$.

Oxygen.-Oxygen was prepared electrolytically and purified as described for hydrogen, with copper oxide substituted for copper in the electric furnace to remove any hydrogen present. Curve $(c)$ is an ionization curve for oxygen taken at a pressure of .002 I mm. The apparatus had been previously used for nitrogen and while the main part of the ionization starts at about 9 volts, traces of ionization begin before this, due probably to small amounts of nitrogen given off from the platinum strip, though the strip had been previously heated to only slightly below its melting point for several hours with the diffusion pump running continually.

There are certain difficulties connected with work in oxygen not experienced in other gases. When pressures of .or $\mathrm{mm}$. or above were used 
no appreciable amount of ionization could be detected even several volts above the ionizing potential of oxygen. This is no doubt the same effect that Franck and Hertz attribute to charged double layers. By using pressures of only a few thousandths of a millimeter this difficulty was avoided. So the curves for oxygen were taken under these conditions.

Mercury Vapor.-Mercury vapor was introduced into the apparatus by removing the freezing mixture from the traps and pumping down to less than .oooor $\mathrm{mm}$. as indicated by the gauge, then allowing the mercury vapor from the various mercury columns to diffuse back into the apparatus.

Franck and Hertz and also Goucher obtained positive electrometer deflections at 4.9 volts in mercury vapor, which they interpreted as ionization.

No ionization could be detected in this experiment below about Io

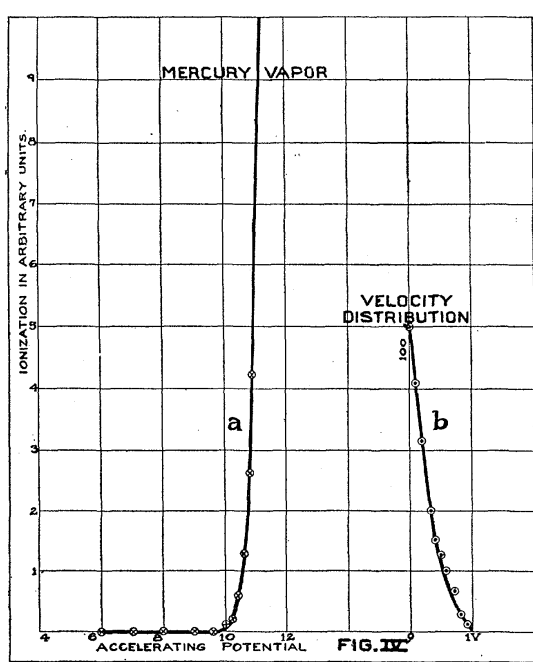

Fig. 4. volts even with electron currents much more intense than those employed in any of the other gases. For this purpose a tungsten wire was used instead of the platinum strip previously employed. Ionization had definitely started at ro volts, but a glance at the corresponding velocity distribution curve (b), Fig. 4, shows that a considerable correction has to be applied to allow for the initial speed of the electrons leaving the wire. For example, the curve shows that the number of electrons which have a velocity corresponding to $(A B$ $+0.5)$ volts is $\mathrm{I} / 5$ as large as the number having a velocity corresponding to $A B$ volts. If this number is sufficient to cause a measurable amount of ionization it would have the effect of pushing the curve half a volt in the direction of greater voltage. So we may safely conclude that the bend in the mercury curve represents the 10.27 type of ionization and that these experiments showed no ionization below this point. This higher value for mercury is in agreement with the result recently obtained by Goucher and Davis and presented at the New York meeting of the American Physical Society on February I7. They showed experimentally that what had been considered ionization occurring at 4.9 volts in mercury vapor was 
really photo-electric effect on the receiving electrode due to radiation from the mercury vapor in the tube which was bombarded by electrons having a velocity between 4.9 and 10.27 volts.

In the apparatus used in this experiment the receiving electrode was of brass slightly oxidized. This was chosen for the ultra-violet light apparatus because it had been tested and found to be very insensitive photo-electrically. The same metal was used in the hot-wire apparatus. This undoubtedly accounts for the fact that no positive deflection of the electrometer occurred below I0.27 volts in mercury vapor as had been found by other investigators. Hence it seems fair to assume, since the present apparatus is not sensitive to photo-electric effect from radiations in the tube, that the results obtained represent true ionization potentials in the other gases also. If this is not the case then certainly the radiation in these other gases is much more intense than in mercury vapor.

The ionization produced by electrons in mercury vapor is very much more intense than it is in the other gases used, that is, after the ionizing potential has been reached a much larger fraction of the collisions result in ionization in mercury than in the other gases. This fact doubtless explains the results obtained for ionizing potentials by Lenard, who found the same value of II volts for all the gases tried. Since the mercury vapor was not frozen out, and since it has been shown that ultra violet light does not give a sufficient supply of electrons for the purpose, no ionization was noticed in any case until the ionizing potential for mercury was reached, which showed itself because of the relatively large number of ions formed in this substance.

Nitrous Oxide.-The nitrous oxide used was taken from a cylinder of nitrous oxide prepared and purified for medical purposes. A large fraction was first allowed to escape from the cylinder and the gas used was taken from the middle of the cylinder.

One of the objects of this experiment was to try a compound, the ionizing potentials of both components of which had been measured separately with the same apparatus, to see how these values would be related to the ionizing potential of the compound. Nitrous oxide seemed to lend itself naturally to this purpose.

The condensation point of this gas being $-92^{\circ} \mathrm{C}$., a freezing mixture of carbon dioxide snow and acetone having a temperature of $-78.2^{\circ} \mathrm{C}$. was substituted for the liquid air. Curve (b), Fig. 3 , is an ionization curve for nitrous oxide. It will be observed that this is almost an exact duplicate of the curve for nitrogen which would indicate that the combination of one atom with another in a compound had no effect on its ionizing potential. Ionization was increasing too rapidly by the time 
the ionizing potential of oxygen was reached to detect any increase in the curve at this point due to oxygen.

Owing to other plans, the investigation had to be terminated before more compounds could be investigated.

The writer wishes here to express his thanks to Professor Zeleny for his suggestion of the general field of investigation as well as to Professors Bumstead and Taylor for their interest and assistance in the work.

\section{Summary.}

I. A comparison has been made of the two methods previously used for determining the ionization potentials of gases by electrons, and the method, where the electrons are liberated by ultra-violet light, is shown to give misleading results because the number of electrons set free is too small. This explains the discrepancy between the results hitherto obtained by this method and those obtained with apparatus where the source of electrons was a hot metal surface.

2. The ionizing potential of several gases has been determined under conditions which tend to minimize the photo-electric effect on the receiving electrode due to radiations in the tube. Results were obtained in good agreement with the accepted values for the following gases: Oxygen 9, nitrogen $7 \cdot 5$, hydrogen II.

3. In hydrogen in addition to this ionization which begins at I I volts, a second and more intense type was found which begins at about 15.7 volts.

4. For mercury vapor no ionization could be detected below I0.27 volts, which is in agreement with the recent work of Goucher and Davis.

5. The ionizing potential of nitrous oxide has been measured and found to be identical with that of nitrogen. It thus appears that in this case at least the ionization potential of nitrogen is not affected by its chemical combination in a compound.

SLOANE LABORATORY,

YALE UNIVERSITY. 